\title{
Fulminant leptospirosis leading to shock lung \& severe coagulopathy in a pregnant patient: A case report and review of the literature
}

\author{
Tharanga $\mathrm{U}^{1}$, Pilapitiya $\mathrm{SD}^{2}$, Senanayake $\mathrm{HMS}^{2}$
}

\begin{abstract}
Background: Leptospirosis is a zoonotic disease with high prevalence in Sri Lanka predominantly in paddy cultivating areas. Severe leptospirosis is rarely described in pregnancy and there is a scarcity of data regarding its effect on pregnant patients. Acute lung injury has been recognized as the commonest fatal complication of leptospirosis. We report a case of delayed presentation of fulminant leptospirosis in a pregnant patient complicated with acute respiratory distress syndrome (ARDS) and severe coagulopathy resulting in death despite the optimum medical care.

Case Presentation: A-31-year-old woman in the twelfth-weekof pregnancy admitted with a subacute history of frequent vomiting, dry cough, watery stools, vaginal spotting, hemoptysis, and progressive shortness of breath. She had tachycardia, hypotension, and acute severe respiratory distress with high-grade fever on admission. Her inflammatory markers were high with evidence of severe coagulopathy. With worsening clinical and biochemical parameters despite intensive medical intervention, patient had a fatal outcome. The autopsy revealed shock lung with multiple intra-alveolar hemorrhages. The serum Leptospira microscopic agglutination test (MAT) was positive confirming the fulminant leptospirosis.

Conclusion: Prolongrespiratory symptoms; positive lung signs with acute respiratory distress can mimic the clinical picture of atypical pneumonia. Delayed presentation of ARDS limits the management options whilst increasing the risk of fatality. A high degree of suspicion is needed to diagnose leptospirosis in pregnancy.

Keywords: Leptospirosis; pregnancy; severe coagulopathy; acute respiratory distress syndrome;shocked lung
\end{abstract}

\section{Introduction}

Leptospirosis is a re-emerging zoonotic disease in Sri Lanka. ${ }^{1}$ Severe leptospirosis is rarely described in pregnancy 2,3 and there is a scarcity of data regarding its effect on the pregnant patients. ${ }^{3}$ Acute lung injury has been recognized as the commonest fatal complication of leptospirosis in certain studies. ${ }^{4}$ Severe leptospirosis in pregnancy may be confused with hemolysis, elevated liver enzymes and low platelets (HELLP) syndrome and acute fatty liver of pregnancy

1. Consultant Resident Physician, Resident Physician Unit, De Soysa Teaching Hospital for Women (DMH), Colombo 08 , Sri Lanka

2. Senior Lecturer and Consultant Physician, Department of Medicine, Faculty of Medicine and Allied Sciences, Rajarata University of Sri Lanka, Saliyapura 50008, Sri Lanka

\section{Corresponding author :}

Umesh Tharanga

Email: desilvaput@gmail.com

(D) https://orcid.org/0000-0001-9669-9941
(AFLP) especially when the patient present in the 3rd trimester. ${ }^{2,3}$ Unusual presentations may delay the diagnosis and subsequently the proper management. Therefore, a high degree of clinical suspicion is vital. Delayed presentation of ARDS in leptospirosis limits the management options and increases the complexity of scenario when it is complicating with severe thrombo-hemorrhagic syndromes. This causes rapid deterioration and unfavorable fatal outcomes. We report a case of delayed presentation of fulminant leptospirosis in the first trimester of pregnancy complicated with acute respiratory distress syndrome and severe coagulopathy ended up with inevitable death despite the optimum medical care.

\section{Case presentation}

A 31-year-old pregnant woman in twelfth week of pregnancy was transferred from the District Hospital, Kakirawa to the gynecology casualty ward, Teaching Hospital Anuradhapura with frequent vomiting for ten days, dry cough for five days, watery stools for two days, vaginal spotting, hemoptysis and progressive shortness of breath (SOB) for a day. She was 
referred to the medical unit for assessment on the following day. There was no history of contact with stagnant and muddy water. She didn't have fever, jaundice, myalgia, abdominal pain, or reduced urine output. On examination pulse rate was 140 beats per minute, blood pressure was $90 / 60 \mathrm{mmHg}$, respiratory rate was 42 cycles per minute and peripheral capillary oxygen saturation ( $\mathrm{SpO} 2$ ) was $80 \%$. The axillary temperature was $380 \mathrm{C}$. The patient was conscious but restless with a severe SOB. There were crepitations in the middle and lower zones of both lungs. Rest of the examination was normal.

Her C reactive protein was very high $(258 \mathrm{mg} / \mathrm{L})$. Full blood count showed high normal white blood count (10.4×109/L) with neutrophil predominant (89\%) low hemoglobin $(9.8 \mathrm{~g} / \mathrm{L})$ and low platelets (127 x 109 /L). Plasma glucose was normal. Serum creatinine, blood urea, AST and ALT were within normal limits. Serum potassium was low (serum K+3.2meq/ L) and serum sodium was in normal range (serum $\mathrm{Na}+141 \mathrm{meq} / \mathrm{L}$ ). Her bilirubin level was high with total bilirubin $29.8 \mathrm{mg} / \mathrm{dl}$ and direct bilirubin 5.8 $\mathrm{mg} / \mathrm{dl}$. A moderate coagulopathy was found with prolonging activated partial thromboplastin time $50.3 \mathrm{sec}(30-46)$ and international normalized ratio 1.45. The D-dimers, serum fibrinogen levels were not done due to un-affordability. Peripheral blood smear showed moderate thrombocytopenia with no evidence of significant red cell fragmentation. Serum creatinine phosphokinase, blood, and urine cultures were normal. The electrocardiogram showed sinus tachycardia (pulse rate of 120 beats/min). The blood gas analysis $(A B G)$ showed type one respiratory failure $\mathrm{pH} 7.314, \mathrm{PaO} 245 \mathrm{mmHg}, \mathrm{PaCO} 232 \mathrm{mmHg}$, HCO3- $18 \mathrm{mmol} / \mathrm{L}, \mathrm{SpO} 2-76 \%$ and acute respiratory distress syndrome (ARDS) (FiO2 40\%, oxygen extraction ratio $112.5 \mathrm{mmHg}$ ). An ultrasound scan showed a single live fetus and normal kidneys, spleen, liver, and biliary tracts. Her two-dimensional echocardiogram was normal.

She was treated at gynecology ward as possible atypical pneumonia with intravenous co-amoxiclav and clarithromycin. After medical review, antibiotics were changed to intravenous meropenem, clarithromycin, and penicillin G. High flow oxygen was given by face mask and hydrated with normal $(0.9 \%)$ saline. The patient was immediately transferred to the medical ward and put on C-PAP (continuous positive airway pressure) and then transferred to the medical intensive care unit (MICU). Two hours after admission to MICU she developed vaginal bleeding and suffered a miscarriage. Her SpO2 dropped to $54 \%$. She was intubated and put on mechanical ventilation. Intravenous sedation, central venous pressure monitoring, restriction of fluid infusion, and continu- ation of intravenous antibiotics were given with maximum supportive care. She developed bleeding from the endotracheal tube and blood pressure dropped to $80 / 50 \mathrm{mmHg}$ subsequently. She was treated with noradrenaline, fresh frozen plasma, tranexamic acid, and vitamin K. Despite of above treatment measures, she developed bradycardia and then the cardio-respiratory arrest. Cardiopulmonary resuscitation (CPR) was unsuccessful and the patient succumbed to death. The autopsy revealed blackish red, rubbery, heavy and edematous shocked lungs in the external appearance (Figure 1) and multiple intra-alveolar hemorrhages with edematous congested lung tissues in the cut section (Figure 2). Small (50ml) pleural effusion on both sides was noted. The serum Leptospira microscopic agglutination test (MAT) was reported as positive (>1/400).

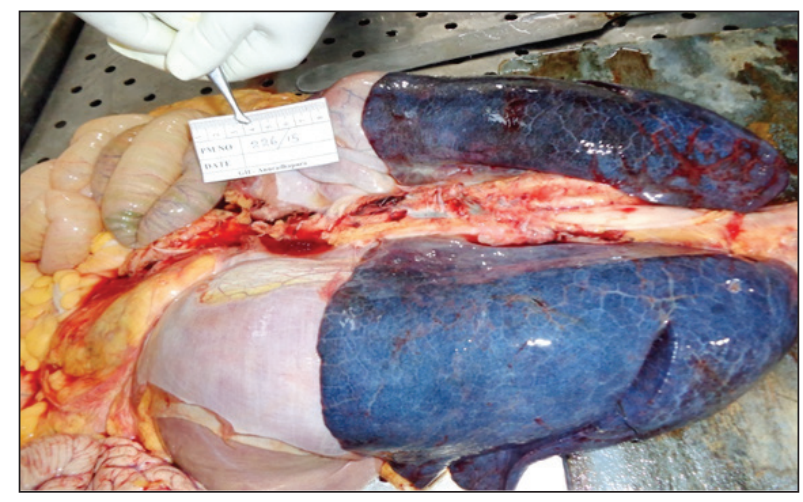

Figure 1: The external appearance of the lungs showed blackish red, rubbery, heavy and edematous shocked lungs.

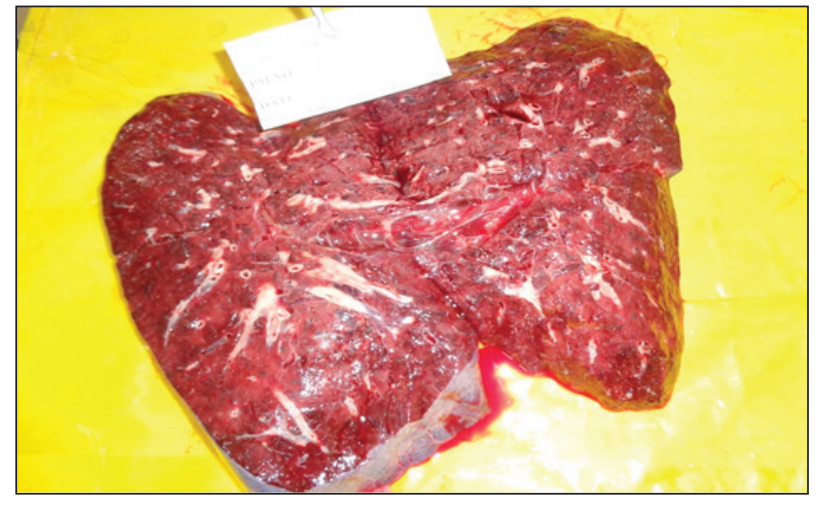

Figure 2: The cut section of a shocked right lung showed multiple intra-alveolar hemorrhages with edematous congested lung tissues.

\section{Discussion}

Leptospirosis has a high prevalence in Sri Lanka predominantly in paddy cultivating areas ${ }^{1}$. There were two reported leptospirosis outbreaks in Anu- 
radhapura district in 2007/08 and 2011/12 according to the regional health directory. ${ }^{5}$ The Leptospira are often transmitted to the humans, especially to the farmers and rice planters by direct exposure of minor skin lesions and mucous membranes to the muddy paddy field which is contaminated by the urine of the reservoir animal. 3,6 Leptospirosis is less reported among the pregnant population in Sri Lanka. This could be due to the minimal exposure to the risk environment during pregnancy. This patient didn't have any recent history of farming, fishing, or contamination with the stagnant and muddy water.The large numbers of animals are included in the carrier profile, importantly rats and mice, dairy cattle, horses, and pigs. They transmit the disease by bacteria which kept alive in their renal tubules.6We believe the probable source of the disease in our patient was through contamination from the urine of rats or domestic animals.

The incubation period may vary from 2-20 days. More than $90 \%$ of symptomatic people have a mild disease: fever, abdominal pain, chills, nausea, vomiting, headache, and myalgia. Conjunctival suffusion is common and can be a useful clinical sign in early recognition. ${ }^{2}$ Our patient had vomiting, dry cough, watery stools for a few days and hemoptysis, vaginal spotting, and breathing difficulty on the day of admission. She did not give a history of fever. Jaundice, myalgia, abdominal pain, or reduced urine output at the time of admission which are the more frequent presentations in leptospirosis. Conjunctival suffusion wasn't observed in our patient. The patients who have oliguria, pulmonary rales, hypotension, and hyperkalemia on admission have a higher mortality. 4,7 Our patient initially had low-marginal blood pressure and positive lung signs without evidence of hyperkalemia or renal impairment which shifted the preliminary management toward the atypical pneumonia.

Leptospirosis causes by the multiple serovars of Leptospira interogans ${ }^{2}$ and can affect multiple organs. It can vary from asymptomatic to acute liver injury, acute kidney injury, pulmonary hemorrhage, myocarditis, rhabdomyolysis, and aseptic meningitis. Leptospirosis has a 5 to $40 \%$ mortality rate in severe disease. $2,3,4$ Thrombocytopenia suggests severe disease and the subsequent risk of severe bleeding. ${ }^{2,6}$ Wagenaar et al. ${ }^{6}$ have reported patients with leptospirosis may develop a spectrum of clinical effects due to infection-associated activation of the coagulation cascade. These include the mild elevation of biochemical tests to severe thrombo-hemorrhagic syndromes such as hemolytic uremic syndrome (HUS), thrombotic thrombocytopenic purpura (TTP), disseminated intravascular coagulation (DIC), and vasculitis. The Patients may present with bleeding, thrombosis or both.The laboratory-hemostatic markers are useful to differentiate these syndromic manifestations. ${ }^{6}$ Our patient developed moderate coagulopathy with clinical and biochemical evidence of disseminated intravascular coagulation (DIC). Initially, she had vaginal spotting, hemoptysis, and subsequently developed vaginal bleeding, inevitable miscarriage, endotracheal bleeding, and hypotension. She had prolonged APTT, INR, low hemoglobin, low platelets, and indirect hyperbilirubinemia.

Incidence of pulmonary manifestation in severe leptospirosis varies from 20 to $70 \% .4$ The clinical manifestations of pulmonary involvement in leptospirosis are cough, breathlessness, hemoptysis, tachypnoea and pulmonary crackles, and wheezes. ${ }^{8}$ Our patient had almost all the features mentioned above. Panaphut et al. ${ }^{4}$ have demonstrated the ARDS and pulmonary hemorrhage were the major complications of leptospirosis, involving $60 \%$ of all-cause mortalityfollowed by acuterenal injury and multiple organ dysfunction (MOD) in their prospective cohort study. Marotto et al. ${ }^{9}$ demonstrated that in ARDS the patient who required mechanical ventilator support associated with 30 to $60 \%$ of mortality. Marc et al. ${ }^{1}$ have described that ARDS happens either direct triggering by leptospires or by their antigenic products affecting pulmonary capillary endothelial cells. In an ABG analysis an acute hypoxemic respiratory failure and oxygen extraction ratio ( $\mathrm{PaO} 2 / \mathrm{FiO} 2$ ratio) $\leq 200$ $\mathrm{mmHg}$ indicates ARDS. ${ }^{8}$ Our patient had evidence of type 1 respiratory failure and severe ARDS. Radiological findings of ARDS include bi-lateral predominantly peripheral, asymmetrical consolidations with air-bronchograms in the CXR and ground-glass opacities involving all lobes, predominantly peripheral lung with dorsal distribution with occasional consolidations and air space nodules in HRCT. ${ }^{1}$ Radiological investigations were not done in our patient due to multiple reasons including the first trimester of pregnancy and rapid deterioration of the clinical condition. The gross autopsy findings of the lungs in ARDS include bilateral, uniformly enlarged, solid airless lungs with increased heaviness. ${ }^{1}$ The cut surface demonstrates the bilateral dry lung with petechial hemorrhages to the pleura and cut surfaces. ${ }^{1}$ Our patient's autopsy revealed blackish red, rubbery, heavy, and edematous shocked lungs with multiple intra-alveolar hemorrhages, edematous congested lung tissues in the cut section which was compatible with typical autopsy findings of ARDS.

Microscopic Agglutination Test (MAT) which is available at the Medical Research Institute (MRI) Colombo, detects both IgG and IgM Leptospira antibodies. 
A laboratory-confirmed case of leptospirosis is defined as, a patient with clinical signs and symptoms consistent with leptospirosis and any one of the following. ${ }^{1}$

1. Four-fold increase in MATtitre in acute and convalescent serum samples;

2. MAT titre $\geq 1: 400$ in single or paired serum samples;

3. Isolation of pathogenic Leptospira species from a normally sterile site;

4. Detection of Leptospira species in clinical samples by histological, histochemical or immuno-staining technique;

5. Pathogenic Leptospira species DNA detected by PCR

Our patient's the serum Leptospira MAT was reported as positive $(>1 / 400)$ and which confirmed the diagnosis of fulminant leptospirosis (Weil's disease). Costa et al. ${ }^{13}$ have reported that the best clinical outcome is expected from the patient who has potentially been treated early. They have also shown that the mixed results have been found in the immune phase of treatment. Doxycycline, ampicillin, or amoxicillin are being widely used in the mild form of the disease, and intravenous penicillin G, cefotaxime, and ceftriaxone are effective in severe leptospirosis. Macrolides, fluoroquinolones, and carbapenems may also be effective. ${ }^{5}$ Our patient presented to us on possibly day 10 of illness with clinically significant pulmonary involvement and coagulopathy with subsequent rapid deterioration within the next 48 hours. This gives an example of delayed presentation of leptospirosis with complications leading to delayed treatment and fatal outcome.

ICU management is mandatory in severe complicated leptospirosis. ${ }^{2}$ These patients need to be managed with careful monitoring of fluid and electroIyte balance. In acute severe renal impairment, may need dialysis. ${ }^{2}$ Transfusion of platelets, packed cells or plasma components should be considered for severely ill patients with DIC and bleeding.8In the case of ARDS, mechanical ventilation is required ${ }^{2}$ Vieira et al. ${ }^{7}$ have reported that corticosteroids, intravenous immunoglobulin (IVIG), and plasma exchange (PLEX) may be beneficial in individuals in whom conventional management fails. ${ }^{7,11,14}$ Herath et al. ${ }^{15}$ also reported that a "possible" better survival rate among the individuals who had been treated with therapeutic PLEX.However, there was no proven effective mode of treatment for ARDS demonstrated in randomized clinical trials up to date. ${ }^{11}$ Corticosteroids were tested with different regimens for ARDS and found inconclusive results. ${ }^{8,16}$ Early corticosteroid usage reduces the requirement of ventilator support. But, there were no reported statistically significant mortality benefits for the patients who are already on mechanical ventilation. Therefore, it has been shown that the use of corticosteroids is only effective when started within the first 24 hours of the onset of pulmonary symptoms. ${ }^{1}$ We didn't use corticosteroids in this patient. Apart from the lack of trial proven benefits, initial confusion with severe atypical pneumonia, rapid deterioration after the admission and ongoing first trimester of pregnancy were the reasons, for not considering corticosteroids in the management of this patient. Though PLEX may be beneficial for some patients with ARDS, it can be hazardous too. It removes coagulation factors and platelets along with offending antibodies and immune complexes leading to dilutional coagulopathy and thrombocytopenia. Gulatiet al. ${ }^{1} 1$ have recommended removing $25 \mathrm{ml} / \mathrm{kg}$ plasma in the selected patients with mild disease. They concluded that patient with severe ARDS doesn't tolerate transient hypoxemia with PLEX for a long period and therefore, may succumb to the death before any obvious benefits of this risky procedure. ${ }^{11}$ As an emergency medical procedure, Extracorporeal membrane oxygenation (ECMO)may support respiratory gas exchange without increasing long term survival in patients with severe ARDS. ${ }^{11}$ However, this procedure is still under evaluation. ECMO gives the best results in an early approach with minimal conventional mechanical ventilation time period. ${ }^{11}$

It has been reported that some confusion in leptospirosis in late pregnancy with hemolysis, elevated liver enzymes, and low platelets (HELLP) syndrome, and acute fatty liver of pregnancy (AFLP) due to similar clinical and laboratory abnormalities. ${ }^{2}$ Gaspari et al. ${ }^{3}$ have described a case of leptospirosis during the third trimester of pregnancy, mimicking the clinical pattern of AFLP or HELLP syndrome. Intrauterine fetal death, abortion, or newborn showing signs of active infection were reported as the possible pregnancy-related outcomes in leptospirosis. ${ }^{2}$

\section{Conclusion}

This case highlights that delayed presentation of leptospirosis in pregnancycould lead to rapidly progressive ARDS and severe DIC with possible death despite the optimum medical care. Prolong respiratory symptoms; positive lung signs with acute respiratory distress can mimic the clinical picture of atypical pneumonia. Delayed presentation limits the management options and increases the risk of fatality. A high degree of suspicion is needed to diagnose leptospirosis in pregnancy. 


\section{Consent}

Written informed consent was obtained from the patient's husband for publication of this case report and any accompanying images.

\section{Conflicts of interests}

The authors declare that they have no conflicts of interest.

\section{Authors' Contributions}

UT and PSD investigated the case. UT and PSD planned hematological, radiological, and other relevant investigations. UT, PSD, and SHMS were involved in forming the case report. UT, SHMS were involved in editing the content of the paper. All the authors read and approved the final version for publication.

\section{Acknowledgments}

The authors would like to thank Dr. Senanayake HMSMK, Consultant Forensic Pathologist who rendered his maximum support during the Postmortem Investigations at Department of Forensic Medicine, Teaching Hospital Anuradhapura, Sri Lanka.

\section{References}

1. Agampodi S. Letters to the Editor: Case definitions in leptospirosis: a note to Sri Lankan researchers. Sri Lanka Journal of Infectious Diseases. 2012; 2(2); 55-57.

2. Hicham S, Ihsane M, Abderahim EB, et al. Multi visceral organ failure related to leptospirosis in a pregnant patient. Indian Journal of Critical Care Medicine 2013; 17(1): 43-45.

3. Gaspari R, Annetta MG, Cavaliere F, et al. Unusual presentation of leptospirosis in the late stage of pregnancy. Journal ofMinerva Anestesiologica 2007; 73(7-8):429-432.

4. Panaphut $\mathrm{T}$, Domrongkitchaiporn $\mathrm{S}$, Thinkamrop B. Prognostic factors of death in leptospirosis: a prospective cohort study in Khon Kaen. Thailand International Journalof Infectious Disease 2002; 6: 52-59.

5. Epidemiological unit 2008 and 2011 circulars to Regional Health Directors of Sri Lanka.http:// epid.gov.Ik/web/attachments/article/179/ epid35122008_eng.pdfhttp://epid.gov.lk/web/attachments/article/179/epi\%2035\%202010.pdf

6. Wagenaar JF, Goris MG, Sakundarno MD, et al. what do coagulation disorders play in the pathogenesis of leptospirosis? Journal ofTropical Medi- cine in Health 2007; 12(1):111-122.

7. Vieira SR, Brauner JS. Leptospirosis as a cause of acute respiratory failure: Clinical features and outcome in 35 critical care patients. Brazilian Journal of Infectious Diseases. 2002; 6:135-9.

8. National guidelines on the management of leptospirosis. Epidemiology unit. Ministry of health, nutrition, and indigenous medicine. Sri Lanka. 2016; 7:28-31.

9. Marotto PCF, Nascimento CMR, Eluf-Neto J, et al. "Acute lung injury in leptospirosis: clinical and laboratory features, outcome, and factors associated with mortality, Journal ofClinical Infectious Diseases. 1999; 29(6):1561-1563.

10. Clavel M, Lheritier G, Weinbreck N, Guerlin A, Dugard $A$, Denes $E$, et al. Leptospirosis: An unusual cause of ARDS. Journal ofCritical Care Research and Practice. 2010; 2010:408365.

11.Gulati S, Gulati A. Pulmonary manifestations of leptospirosis. Journal ofLung India. 2012; 29(4):347-353.

12. Guy N. Rutty Essentials of Autopsy Practice: Recent Advances, Topics and Developments. Springer Science \& Business Media. 2003; 95.

13. Costa $E$, Lopes AA, Scramento $E$, et al. Penicillin at the late stage of leptospirosis: a randomized control trial. Journal of the Sao Paulo Institute of Tropical Medicine 2003; 45(3):141-145.

14.Shenoy VV, Nagar VS, Chowdhury AA, Bhalgat PS, and Juvale NI, "Pulmonary leptospirosis: excellent response to bolus methylprednisolone," Postgraduate Medical Journal. 2006; 82: 971:602-606.15. Herath N, Uluwattage W, Weliwitiya T, et al. Sequel and therapeutic modalities of leptospirosis associated severe pulmonary haemorrhagic syndrome (SPHS); a Sri Lankan experience. Journal of BioMed Central Infectious Diseases. 2019; 19: 451.

16. Villar J, Ferrando C, Martínez D, et al. Dexamethasone treatment for the acute respiratory distress syndrome: a multicenter, randomized controlled trial. Lancet Respiratory Medicine. 2020; 8(3):267276. 